\title{
Improved Measurement of Three-Dimensional Magnetic Properties of SMC Material
}

\author{
Yongjian $\mathrm{Li}^{1,2}$, Qingxin Yang ${ }^{1}$, Youhua Wang ${ }^{1}$, \\ Jingfeng Sun ${ }^{1}$ \\ Province-Ministry Joint Key Laboratory of \\ Electromagnetic Field and Electrical Apparatus Reliability \\ Hebei University of Technology Tianjin , China \\ yoli@eng.uts.edu.au
}

\author{
Jianguo Zhu', Youguang Guo ${ }^{2}$, Zhiwei Lin $^{2}$, Wei Xu ${ }^{2}$ \\ Yi wang ${ }^{2}$ \\ School of Electrical, Mechanical and Mechatronic Systems \\ University of Technology \\ Sydney, NSW 2007, Australia \\ joe@eng.uts.edu.au
}

\begin{abstract}
An improved three-dimensional (3-D) magnetic property measurement system, which can indirectly measure the magnetic field strength $H$ vector in a cubic sample of soft magnetic composite (SMC) material by controlling the three components of the magnetic flux density $B$ vectors, has been developed and calibrated. This paper especially describes the improved structure of sample with sensing $H$ and $B$ coils, and deals with the magnetic compensation problem. The relationship between the $B$ vector and $H$ vector has been measured under alternating and rotating flux conditions by using the 3-D magnetic measuring system, and the core loss features have been analyzed when the $B$ loci are controlled as circles with increasing magnitudes and ellipses evolving from a straight line to circle in three orthogonal planes. (Abstract)
\end{abstract}

Keywords-soft magnetic composite; 3-D tester; isotropy; compensation; calibration; rotational magnetic property (key words)

\section{INTRODUCTION}

Soft magnetic composite (SMC) material is widely applied to 3-D magnetic flux machines. Conventional methods for detecting the magnetic properties of SMC material are not accurate due to the rotational magnetic domains, as the magnetic properties of the materials with 3-D magnetic fluxes must be considered [1]. By using a novel 3-D magnetic properties tester developed in University of Technology, Sydney (UTS), a kind of SMC material, SOMALOYTM 500, made of highly pure iron powder with surface coating to ensure low eddy current loss, has been measured and analyzed [2]. A cubic sample with the side length of $22 \mathrm{~mm}$ and six guard pieces of $22 \times 22 \times 5 \mathrm{~mm}^{3}$ were cut from a large cylindrical perform. In this paper, in order to obtain more accurate experimental results, a new structure of the sample with $\mathbf{H}$ and $\mathbf{B}$ sensing coils has been designed and constructed. The process for fabricating the improved sensing coils and the analysis method of magnetic compensation are described. Furthermore, a series of real 3-D hysteresis loci under various experimental conditions are presented [3].

\section{STRUCTURE OF THE 3-D TESTING System}

The UTS 3-D magnetic property testing system consists of a 3-D yoke to guide the magnetic fluxes along the orthogonal $x$-, $y$-, and $z$-axis, a data acquisition system, three groups of excitation coils which are wound around the legs of the 3-D yoke, and a feedback control system comprising a control unit and three high power amplifiers [2,3]. Three groups of computer controlled excitation coils are used to produce magnetic fields in three axes, so that various flux density loci, such as circularly and elliptically rotating flux density vectors, can be obtained. The components of the sample surface field intensity $\mathbf{H}$ are measured by 12 sensing coils and the flux density $\mathbf{B}$ components are measured by three search coils wrapped around the cubic sample. Fig.1 illustrates the cross section of the 3-D tester core.

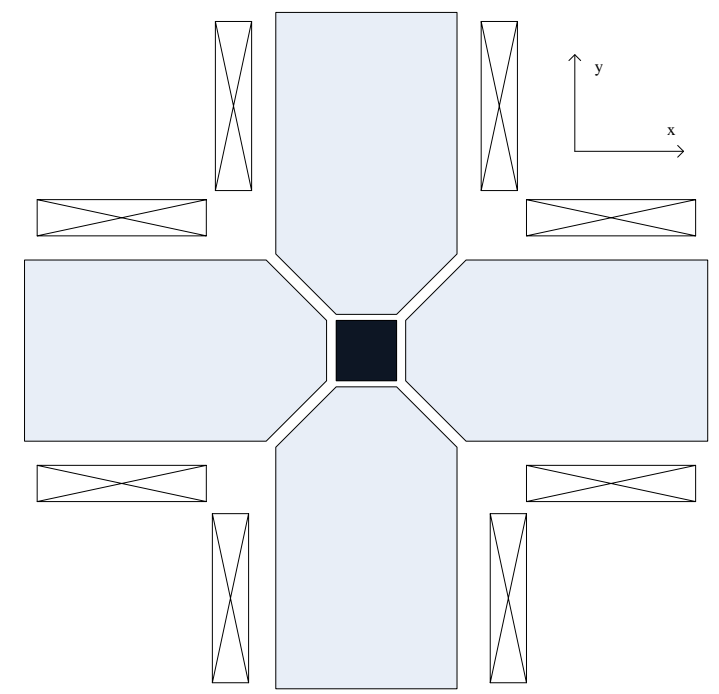

Figure 1. Cross section of the 3-D tester core (skimmed the $z$ direction)

\section{IMPROVEMENT OF THE TESTING SYSTEM}

\section{A. Improved Structure of SMC Sample with Sensing Coils}

The original tester structure can be improved by considering that the surface area of the $\mathbf{H}$ coil equals that of a side of the cubic sample and the magnetic field in the sensing area may not be uniform. An improved structure is proposed as following. Epoxy resin perform is cut to be the shape as shown in Fig.2 (a). The thickness is ground to be about

This paper is sponsored by the Science Foundation Projects of Hebei Province, China , "Three Dimensional Rotating Electromagnetic Field Numerical Analysis Based on Magnetic Reluctivity Tensor”, the project number is E2008000051. 
$0.55 \mathrm{~mm}$, and the height of the embedded B coil is $0.5 \mathrm{~mm}$, so the circle in the center of the epoxy resin can hold the $\mathbf{B}$ coil. $\mathbf{H}$ sensing coils are wound and attached to the surface of epoxy base which impinge on the surface of SMC sample. So the minimized $\mathbf{H}$ coil and the embedded $\mathbf{B}$ coil can be attached to the central area of each side of the cubic sample, as shown in Fig. 2(b). Six sets of combined $\mathbf{H}$ and $\mathbf{B}$ coils are placed on the six sides of the cubic sample in given order and the coils on the opposite sides are connected in series. It has been found the field near the central area is quite uniform. The tangential component of $\mathbf{H}$ is continuous if no applied current flows on the sample surface. The sensing area is now centralized and the gap between the sample and $\mathbf{H}$ coil is contracted. This new structure of sensing coils can improve the precision of measurement. Both the $\mathbf{H}$ coils and the $\mathbf{B}$ coils are attached to the surface of sample. It also simplifies the fixing work, so samples can be changed easily.

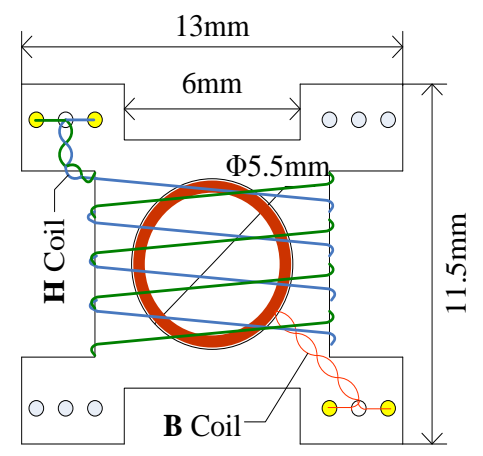

(a) H coil and B coil

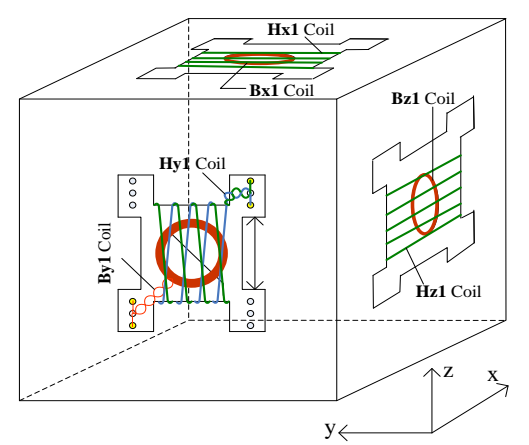

(b) Sample with sensing coils

Figure 2. Improved structure of the tester core

\section{B. Magnetic Compensation}

For obtaining ideal testing data, magnetic compensation should be considered in several aspects. Firstly we should reduce the influence caused by the imperfect winding, which can form some effective areas on the face and the side face of coils where the fluxes can go through and an additional electromotive force (emf) can be induced in the coil. The double-cross-winding structure of $\mathbf{H}$ sensing coil is adopted, as shown in Fig. 2(a), The two layers can induce signals with equal magnitude but opposite direction, and hence cancel each other. Furthermore, the method of wire wrapping is adopted, which can shrink the area of the circle and optimize the magnetic circuit. Secondly, the adoption of the guard pieces which are the same material as the sample can significantly decrease the equivalent reluctance of the magnetic circuit and hence the excitation current required to magnetizing the sample. Finally, the measured $\mathbf{B}$ and $\mathbf{H}$ components are not the true values if the axes of the sensing coils are misaligned with the axes of the excitation coils. This error can be corrected by using the rotational transformation of coordinates [4].

\section{Calibration of the Sensing Coils}

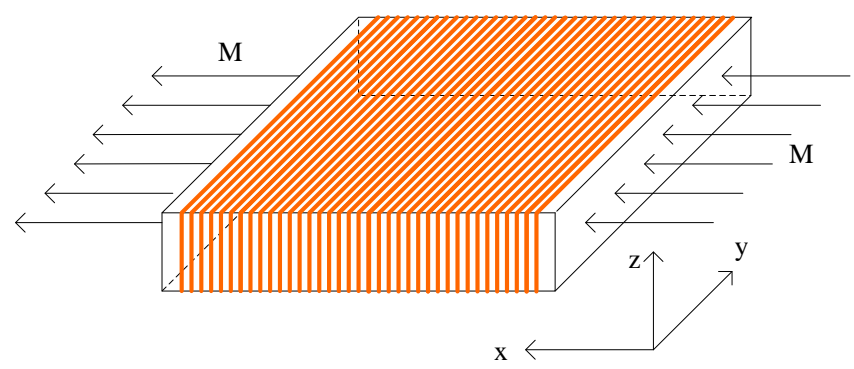

Figure 3. Magnetic line of force through the coil

In Fig.4, the magnetic line of force passes through the windings effective area in $x$ direction, inductive electromagnetic force will be generated due to Faraday law of electromagnetic induction.

$$
E=-N \frac{\mathrm{d} \phi}{\mathrm{d} t}
$$

The calibration of the improved $\mathbf{H}$ and $\mathbf{B}$ coils in $x$ direction is performed in a long solenoid, which can generate a uniform magnetic field. The surface of the coil is set to be parallel to the generated field [2]. The coefficients are calculated by

$$
\begin{gathered}
K_{H}=\frac{V_{H}}{\sqrt{2} \pi f \mu_{0} H_{m}} \\
K_{B_{i}}=N_{B_{i}} A_{s p}
\end{gathered}
$$

where $V_{H}$ is the rms value of the induced emf, $\mu_{0} H_{m}$ the peak value of the air flux density in the middle of the solenoid, $f$ the excitation frequency, $N_{B i}$ the number of turns of the $B_{i}$ coil, and Asp the cross-sectional area of the sample. The final results, which were obtained by averaging over a number of measurements, are $K_{H x}=0.0021743, \quad K_{H y}=0.0021589$, $K_{H z}=0.002265\left(\mathrm{~m}^{2}\right) ; \quad K_{B x}=0.0018015, \quad K_{B y}=0.0018024$, $K_{B z}=0.0018893\left(\mathrm{~m}^{2}\right)$.

In intense magnetic field, the influence in the other two directions is obvious and should be concerned. A new method of calibrating the $\mathbf{H}$ coil's coefficient in $z$ direction is by means of the embedded $\mathbf{B}$ coil which is calibrated in the solenoid. Similarly, the $\mathbf{B}$ coil's coefficient in parallel direction can be obtained by means of $\mathbf{H}$ coil. This calibration job must be carry out in the 3-D tester. Table Ishows the calibration data in the 3-D tester. The $50 \mathrm{~Hz}$ alternating current signal comes from a signal generation and power amplifier. The averaging measurement results of the $\mathbf{H}$ coils' coefficient in $y$ and $z$ directions are $K_{H}^{y}=8.815 \times 10^{-7} K_{H}^{z}=6.229 \times 10^{-5}\left(\mathrm{~m}^{2}\right)$. The 
average coefficient of the $\mathbf{B}$ coils' in parallel direction is $K_{B}^{p}=1.242 \times 10^{-5}\left(\mathrm{~m}^{2}\right)$.

TABLE I. CALIBRATION DATA IN 3-D TESTER

\begin{tabular}{|c|c|c|c|c|c|c|c|c|}
\hline $\mathbf{i}(\mathbf{A})$ & $\mathbf{u}_{\mathbf{B} 1}(\mathbf{m V})$ & $\mathbf{u}_{\mathbf{H 1}}(\mathbf{m V})$ & $\mathbf{B}_{\mathbf{B} 1}(\mathbf{m T})$ & $\mathbf{K}_{\mathbf{H 1 z}}$ & $\mathbf{u}_{\mathbf{H} \mathbf{2}}(\mathbf{m V})$ & $\mathbf{u}_{\mathbf{B} 2 \mathbf{p}}(\mathbf{m V})$ & $\mathbf{B}_{\mathbf{H} 2}(\mathbf{m T})$ & $\mathbf{K}_{\mathbf{B} 2 \mathbf{p}}$ \\
\hline 0.9 & 8.13 & 0.25 & 14.35806 & $5.542 \mathrm{E}-05$ & 7.19 & 0.04 & 10.279332 & $1.239 \mathrm{E}-05$ \\
\hline 1.453 & 13.17 & 0.44 & 23.259 & $6.022 \mathrm{E}-05$ & 11.54 & 0.06 & 16.4984 & $1.158 \mathrm{E}-05$ \\
\hline 1.915 & 17.38 & 0.59 & 30.69411 & $6.119 \mathrm{E}-05$ & 15.22 & 0.08 & 21.759588 & $1.17 \mathrm{E}-05$ \\
\hline 2.665 & 24.23 & 0.84 & 42.79161 & $6.248 \mathrm{E}-05$ & 21.55 & 0.12 & 30.809404 & $1.24 \mathrm{E}-05$ \\
\hline 3.541 & 32.26 & 1.18 & 56.97306 & $6.593 \mathrm{E}-05$ & 28.71 & 0.16 & 41.045846 & $1.241 \mathrm{E}-05$ \\
\hline 4.108 & 37.48 & 1.32 & 66.19189 & $6.348 \mathrm{E}-05$ & 33.35 & 0.185 & 47.679518 & $1.235 \mathrm{E}-05$ \\
\hline 4.748 & 43.34 & 1.52 & 76.541 & $6.321 \mathrm{E}-05$ & 38.76 & 0.22 & 55.414036 & $1.264 \mathrm{E}-05$ \\
\hline 5.137 & 46.9 & 1.66 & 82.82817 & $6.379 \mathrm{E}-05$ & 41.83 & 0.24 & 59.803126 & $1.277 \mathrm{E}-05$ \\
\hline 5.559 & 50.73 & 1.79 & 89.59218 & $6.36 \mathrm{E}-05$ & 45.2 & 0.26 & 64.621116 & $1.281 \mathrm{E}-05$ \\
\hline 6.056 & 55.29 & 1.95 & 97.6454 & $6.357 \mathrm{E}-05$ & 49.25 & 0.29 & 70.411282 & $1.311 \mathrm{E}-05$ \\
\hline
\end{tabular}

When the cubic SMC sample is placed into the sensing house and subjected to a magnetic field, the voltage for the $x, y, z$ directions in $\mathbf{H}$ coils $U_{H}$ and $\mathbf{B}$ coils $U_{B}$ can be expressed as

$$
\begin{aligned}
& \left\{\begin{array}{l}
U_{H x}=\mu_{0}\left(K_{x}^{x} \frac{\mathrm{d} H_{x}}{\mathrm{~d} t}+K_{x}^{y} \frac{\mathrm{d} H_{y}}{\mathrm{~d} t}+\frac{K_{x}^{z}}{\mu_{0}} \frac{\mathrm{d} B_{z}}{\mathrm{~d} t}\right) \\
U_{H y}=\mu_{0}\left(\frac{K_{x}^{y}}{\mu_{0}} \frac{\mathrm{d} B_{x}}{\mathrm{~d} t}+K_{y}^{y} \frac{\mathrm{d} H_{y}}{\mathrm{~d} t}+K_{y}^{z} \frac{\mathrm{d} H_{z}}{\mathrm{~d} t}\right) \\
U_{H z}=\mu_{0}\left(K_{z}^{x} \frac{\mathrm{d} H_{x}}{\mathrm{~d} t}+\frac{K_{y}^{z}}{\mu_{0}} \frac{\mathrm{d} B_{y}}{\mathrm{~d} t}+K_{z}^{z} \frac{\mathrm{d} H_{z}}{\mathrm{~d} t}\right)
\end{array}\right. \\
& \left\{\begin{array}{l}
U_{B x}=K_{B z}^{z} \frac{\mathrm{d} B_{z}}{\mathrm{~d} t}+\mu_{0} K_{B z}^{p} \frac{\mathrm{d} H_{x}}{\mathrm{~d} t} \\
U_{B y}=K_{B x}^{x} \frac{\mathrm{d} B_{Z}}{\mathrm{~d} t}+\mu_{0} K_{B x}^{p} \frac{\mathrm{d} H_{y}}{\mathrm{~d} t} \\
U_{B z}=K_{B y}^{y} \frac{\mathrm{d} B_{z}}{\mathrm{~d} t}+\mu_{0} K_{B y}^{p} \frac{\mathrm{d} H_{z}}{\mathrm{~d} t}
\end{array}\right.
\end{aligned}
$$

Actually the coefficients can be written in matrix form. Then the field strength and flux density components can be worked out by solving matrix equations.

\section{EXPERIMENTAL RESULTS}

Fig.4 shows the curve of magnetic flux densities follows the increasing alternating current in the center of the 3-D tester without sample. It denotes that the linearity of the B curve is nearly perfect. Uniformity of the magnetic field is to ensure accurate measurement.

Fig.5 plots the hysteresis loops and core losses when the $\mathbf{B}$ is controlled to be sinusoidal alternating at $50 \mathrm{~Hz}$ along the $x, y$, and $z$-axis, respectively. It is noticed that the loops for the $x$ and $\mathrm{z}$-axes are similar, while the $\mathrm{y}$-axis seems to be the easy axis, though the sample is expected to be magnetically isotropic. The permeability along the $y$-axis is higher than that along the $x$ or $z$-axis. The reason is the difference of mass densities of the SMC sample in the compressing and perpendicular directions. The preform was compacted in a cylindrical die with a high pressure along the $y$-axis, i.e. the axis of the cylinder [5].

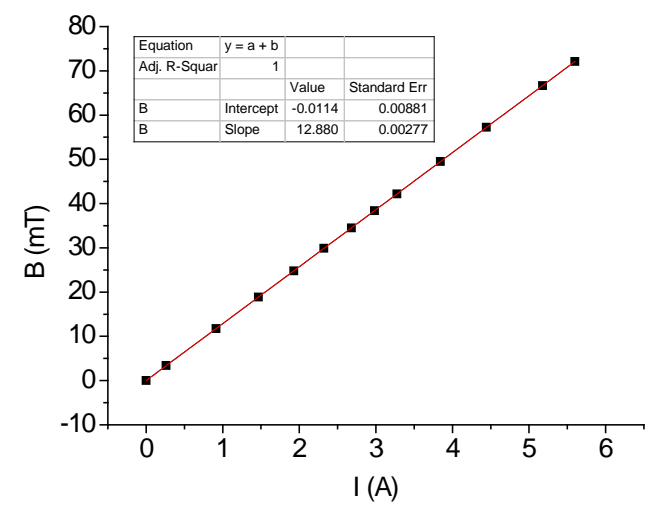

Figure 4. Magnetic flux densities in the center of 3-D testee without sample

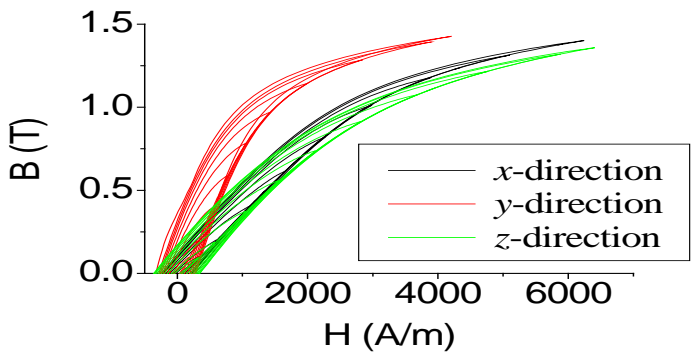

(a) Hysteresis loops

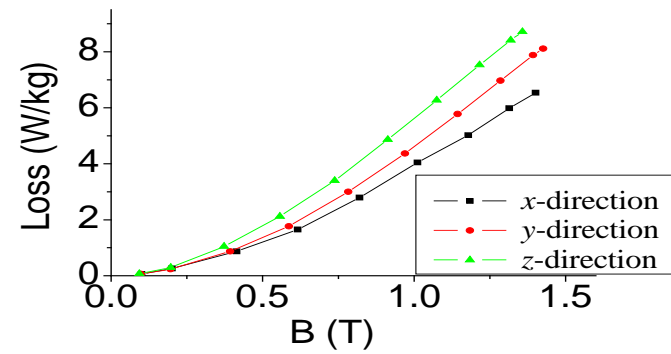

(b) Core losses 
Figure 5. Hysteresis loops and core losses for alternatin magnetizations on the $x-, y$-, and $z$-axis

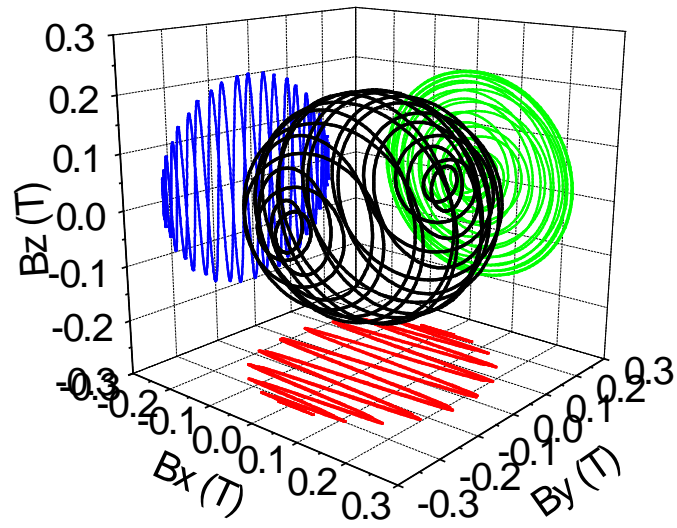

(a) Spherical B locus

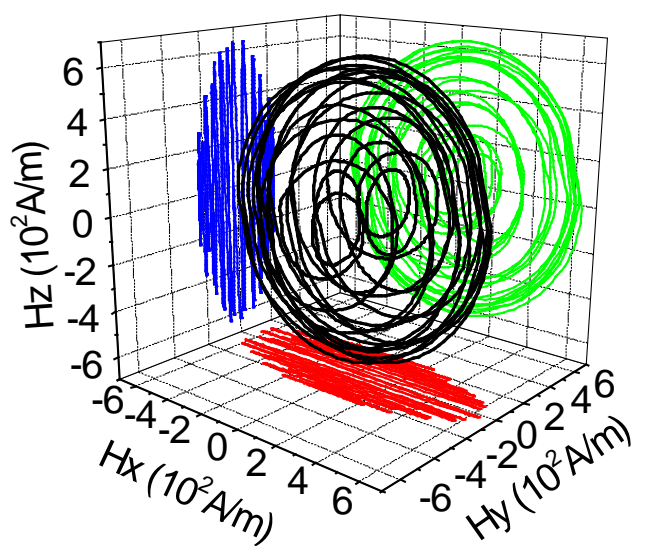

(a) $\mathbf{H}$ locus

Figure 6. Spherical $\mathbf{B}$ locus and corresponding $\mathbf{H}$ locus

Fig.6 shows loci of $\mathbf{B}$ vector and corresponding $\mathbf{H}$ vector in the sample when the $\mathbf{B}$ locus is controlled to be spherical. The diameter of the sphere is $0.4 \mathrm{~T}$. The frequency of $\mathrm{B}_{\mathrm{y}}$ is set at 20 $\mathrm{Hz}$ while that of $B_{x}$ and $B_{z}$ is set at $400 \mathrm{~Hz}$. The envelope of $B_{x}$ and $B_{z}$ is at $20 \mathrm{~Hz}$. It can be seen that the $\mathbf{H}$ locus is measured appropriately when $\mathbf{B}$ locus is well controlled. Analysis of the projection images in three planes is in accord with corresponding view as in [6,7]. It is evident that the 3-D magnetic property testing system work very well.

Fig. 7 plots the power losses when the elliptical $\mathbf{B}$ loci lie in the three planes, with the major axis fixed at $1.3 \mathrm{~T}$. For example, when the $\mathbf{B}$ locus approaches to be a circle, the core losses are $7.4 \mathrm{~W} / \mathrm{kg}, 14.1 \mathrm{~W} / \mathrm{kg}$ and $12.3 \mathrm{~W} / \mathrm{kg}$ in the $x o y-$, yoz-, and zox-plane, respectively. However, the losses depend strongly on the orientation of major axis and the axis ratio when the $\mathbf{B}$ locus is elliptical.

\section{DISCUSSION AND CONCLUSION}

$\mathbf{B}$ and $\mathbf{H}$ sensing coils are reconstructed and calibrated in long solenoid and 3-D tester. The detailed calibration outcome testified that the non-virtual direction of the coils can induct emf under the intense magnetic field though some valid compensation methods are concerned. The coefficients of nonvirtual directions are tested and quantified can help to improve the testing precision. By using the improved 3-D magnetic tester, the magnetic properties of an SMC sample have been systematically measured. It is found that the $\mathbf{B}$ and $\mathbf{H}$ loci lie in the same magnetization plane, but the $\mathbf{H}$ loci and power losses strongly depend on the orientation, position, and process of magnetization. Under the different magnetizing conditions, experimental data would be more accurate thanks to the improved sensing coils.

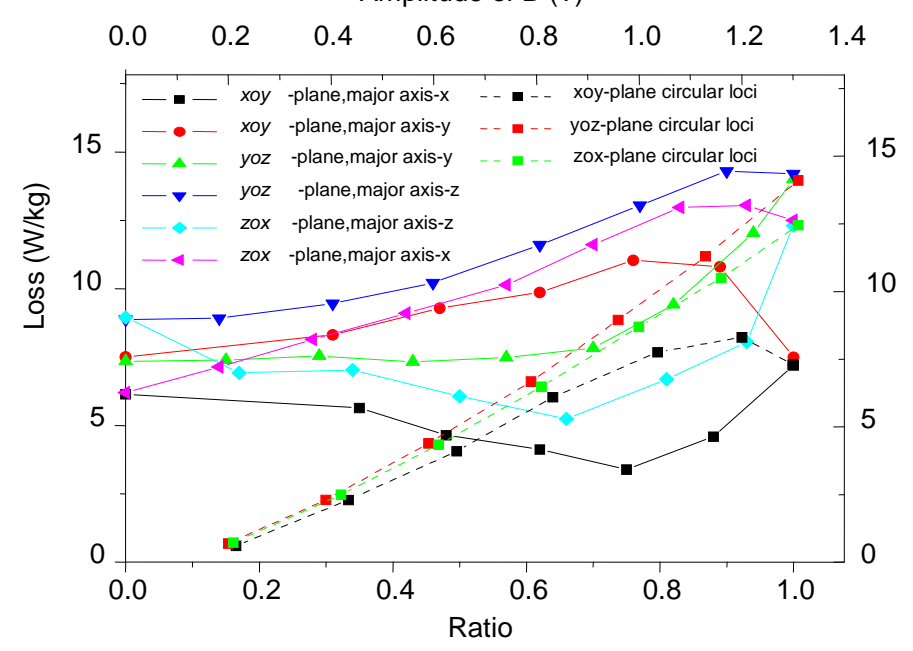

Figure 7. Power losses vs ratios of minor axis to major axis (bottom axis) of elliptical B loci evolving from lines to circles via ellipses, and versus amplitudes of circular B loci (upper axis).

\section{REFERENCES}

[1] Y.G. Guo, J.G. Zhu, P.A. Watterson and W. Wu, "Comparative study of 3D flux electrical machines with soft magnetic composite core," IEEE Trans. on Industry Application, 39(6):1696-1703, 2003.

[2] J.G. Zhu, J.J. Zhong, Z.W. Lin, J.D. Sievert, "Measurement of magnetic properties under 3-D magnetic excitations," IEEE Trans. on Magnetics, 39(5):3429-3431, 2003.

[3] Z.W. Lin, J.G. Zhu, and Y.G. Guo, "Three-dimensional hysteresis of soft magnetic composite,” J. Appl. Phys. 99:08D909, 2006.

[4] J.J. Zhong, Y.G. Guo, J.G. Zhu and Z.W. Lin, "Characteristics of soft magnetic composite material under rotating magnetic fluxes,” J.Magn. Magn. Mater., 299 (2006) 29-34.

[5] Y.G. Guo, J.G. Zhu, Z.W. Lin, J.J. Zhong, H.Y. Lu, and S.H. Wang, "Determination of 3-D magnetic reluctivity tensor of soft magnetic composite materials,” J. Magn. Magn. Mater., 312:458-463, 2007.

[6] Y.G. Guo, J.G. Zhu, Z.W. Lin, and J.J. Zhong, “3D vector magnetic properties of soft magnetic composite material,” J. Magn. Magn. Mater., 302 (2) (2006) 511.

[7] Z.W. Lin, J.G. Zhu, and Y.G. Guo, "Three-dimensional magnetic properties of soft magnetic composite materials,” J. Magn. Magn. Mater., 312:158-163,2007. 